\title{
The Melding of Form and Function
}

\author{
Paul Winkelman \\ University of British University \\ pwinkel@mech.ubc.ca
}

\begin{abstract}
The engineering design process is traditionally modelled as proceeding from the functional domain to the physical domain. Function thus represents more abstract concepts and the form or structure resulting from the design process as the more concrete. A comparison of mechanical design with process planning, however, reveals that this model does not apply to all areas of engineering design. In mechanical design, engineers select those forms they believe will perform the desired function; in process planning, engineers select those functions (i.e., machining operations) they believe will create the desired form. This reversal of form and function raises important issues concerning how engineering design should be modelled as form and function may not be as distinct as the traditional model of engineering design might suggest. This paper is an initial exploration to better understand the nature and the implications of this "reversibility". Potential benefactors of this research are computerized design tools which use form and function to aid designers. By building upon a framework which supports both the mechanical design and the process planning perspectives, the design tool promises to better serve the needs of its users.
\end{abstract}

\section{Introduction}

Design can be described as "a transformation from the functional domain to the physical domain" (p. 144 [1]). Although one may question the validity of this definition for all areas of design, it does describe much of engineering design quite well. Mechanical design is a case in point where engineers begin with functional requirements and transform these requirements into a physical embodiment. Alternately, we might state that mechanical design is the process of selecting or creating forms that will produce the desired function(s). The basic direction of the design process is from function to form. It is the function realized by of that final form that is the ultimate goal of the design process.

Upon closer inspection, however, this definition breaks down even in the engineering domain. Consider process planning in manufacturing engineering. Process planners begin with requirements of form and determine which machining operations will result in that form. One might say that they select or create functions the will produce the desired form(s). The basic direction of process planning is from form to function. It is the form of the final product that is the ultimate goal of process planning. Indeed, process planners may have no knowledge of the functions of the forms they create.

Thus, mechanical engineering and manufacturing engineering carry out their design activities, at least in certain times, in opposite directions. Nevertheless, it is the direction of mechanical design that has dominated the engineering concept of design. This is perhaps somewhat ironic as much of the motivation for the development of design concepts within mechanical engineering would seem to have come from a desire to make designed products of mechanical engineers easier to manufacture.

Historically, process planning was seen as a separate activity from engineering design. Designs were "tossed over the wall" (p. 190, [2]) meaning that the designing engineers saw production considerations as falling outside their area of responsibility. The "tossing over the wall" may also be interpreted as representative of a hierarchy: the designers were the professional engineers and those that manufactured the product were technicians with little or no professional status. The hierarchical structure prevented process planning from being seen as a bona fide design activity. The "real" design activities took place on the other side of the "wall".

The concept of concurrent engineering sought to tear down the "wall" and bring the designers and manufacturers (among others) closer together. The design of the product would no longer be divorced 
from its manufacture. With mechanical design and process planning being part of the same team, it becomes increasingly difficult to view manufacturing as a non-design activity. Indeed, the very existence of "manufacturing engineering" implies a strong design component within manufacturing. Unfortunately, its status is still lagging behind the more traditional mechanical engineering as indicated by the continued domination of the function-to-form concept of design. Part of the reason may be that at the end of the design process, what one sees is the finished product, i.e., the form. The output appears to be the same for both mechanical design and process planning.

For the purposes of this paper, I consider process planning to be a legitimate engineering design activity. Thus, engineering design can therefore be viewed as a function-to-form or a form-to-function process. This view weakens the distinction between form and function and suggests that a type of "interchangeability" or "reversibility" may be possible.

\section{Objectives}

The objectives of this research are, first, to explore form and function by examining the concepts that have been developed around them and how one is understood in the light of the other; second, to find ways that form and function might be brought closer together and explore the implications of such a melding; and, third, to investigate the implications of these findings as it relates to the development of design tools, particularly those tools that are computer-based that draw on some link between form and function as a means of assisting the designing engineer (such as [3]).

\section{The Meanings of Form}

Any discussion of form and function requires a definition of these terms. I have already alluded to some possible definitions of form. Perhaps the dominant concept of form is that of a physical product. Images of a car, a bolt, and a generator readily come to mind as engineering examples. These engineering products have definite tactile qualities associated with them. They also have symbolic qualities and these are often related to aesthetic qualities. Within the engineering domain, however, the physical aspects of the products are of much greater interest.

The advent of the computer has forced engineers to reconsider whether an engineering product should be confined to a physical object. Software constitutes an important engineering product, yet it has no physical form, being wholly symbolic. Being wholly symbolic, however, has not rendered software wholly aesthetic: its aesthetics lies in how some of those symbols are brought together, such as in a clever algorithm.

Another product of engineering design is that of the process, such as the development of a chemical, biological or manufacturing process. As I suggested earlier, however, the process bears greater resemblance to a function than a form as the output of that process is the form, i.e., a chemical compound, a biological substance or a machined component.

Thus, computer software alerts us to the problem of assuming that engineering products take on physical form; and the engineering process reminds us that not all engineering products should be designated as "form". Given the range of possible interpretations, I suggest any definition of form within the engineering domain will remain slippery and elusive. However, to avoid confusion, the discussions which follow will favour the "physical" definition. I use the narrow definition to show how that traditional concept might expand.

\section{Form Follows...}

To further elucidate the possible meanings of form, I wish to examine what it is that gives rise to a particular form as opposed to some other form.

\subsection{Form Follows Function}

If the average person on the street were asked to discuss form and function, the axiom of "form follows function" would likely come to mind. For many, this speaks of some virtue which designers should strive for. But what does it actually mean?

Viewing engineering design as a logical process, "form follows function" may allude to a necessary, logical connection between the two. In other words, once the functional requirements have been specified, the form the product must take becomes self-evident. The validity of such logic is questionable as there are several forms that can fulfill a given functional requirement. A bolt and a rivet are two distinct forms, yet both can perform the function fastening. It is conceivable that, by precisely stating all the functional requirements, forms can be unambiguously specified. At this point, however, one would no longer feel compelled to call this process design.

Perhaps 'form follows function' can be understood differently. The quote is attributed to Louis Sullivan, a U.S. architect [4]. Sullivan's intent was not to insist on a logical reasoning from function to form, but rather to remind designers to address functional requirements when creating the form. As Alexander [5] puts it, "form responds to function" (p. 1, emphasis mine). Evidentially, form was following or responding to something else. What might that something else have been? 


\subsection{Form Follows Precedent}

If designers need to be reminded that "form follows function", perhaps that is because all too often "form follows precedent" [6]. Viewed in a negative light, "form follows precedent" speaks of design as honouring tradition while ignoring much needed functional attributes.

In a more positive light, one might argue that the traditional way is the functional way. The traditional forms are what they are because other forms have been tried and were not found to offer any improvement. To change the traditional form is to play with fire.

The idea that form follows precedent closely resembles design in what Alexander [5] calls an unselfconscious process. In unselfconscious process, forms are created according to the tradition that preceded it. People learn form-making by repeating a single, familiar pattern. and designers learn by habit, repetition and practice. There is a right and a wrong way to build but no guiding principles. When problems arise, there are remedies in place to fix the problems, but the builders do not ponder other options; the response is automatic. The construction of the form is passed on from one generation to the next and no written record of the building process is left.

The forms that result from the unselfconscious process exhibit "clarity and good fit". As an example, Alexander describes the mousgoum hut of tribespeople in northern Cameroon. These huts have been built for many centuries, display little variation and are constructed of materials that are locally available and familiar to the builders. These mud huts are hemispherical and this shape minimizes heat transfer in the hot sun. The shape is maintained by vertical wooden ribs. These ribs also provide guideways for rainwater as well as a scaffold-like structure for building and later repairing the hut. The huts themselves are grouped together with the chief's hut in the middle. This grouping maintains a social order and offers protection against wild beasts and invaders.

Alexander's account suggests that the axiom "form follows precedent" leads to desirable, well-adapted forms. However, this process does not explain how the basic form of the hut came into existence for perhaps many design options were considered before the people settled on the present form. The success of the process also assumes that the environment is relatively stable. If the environment should suddenly change, builders may have great difficulty responding to the new situation. Alexander gives the example of Slovakian peasants who were famous for the beautiful shawls they made. However, when presented with new dyes and a wider range of colours, the shawls lost their beauty. The shawlmakers, steeped in tradition, were unable to adapt to the new dyes.

\subsection{Form Follows Fabrication}

Bill [7] points out that it was often considered normal that the form of a product reflect the way it was fabricated. This comment presumably arose as it was a concern at the time of writing (1952). When mass-produced, machine-fabricated merchandise first began to flood the market, hand-fabricated products were shunned for they were deemed sub-standard, out of date and maybe even ugly. To be modern, one had to buy the mass-produced products with their smooth, well defined forms. As a simple example, consider bread. Mass-produced bread has an easily recognizable form, with mostly straight sides and bulging, round tops. Misshapen loaves are readily assumed to be home-made. Not too long ago, consumers of such deviant loaves were assumed to be out of step with modern times.

In more recent times, hand-made products have regained at least some of their status. They perhaps have been assigned some symbolic value, reminding us with fondness of some bygone days. They also speak of a uniqueness and a labour of love in contrast to the ubiquity of the manufactured product produced by an indifferent machine.

\subsection{Form Follows Failure}

The idea that "form follows failure" was championed by Petroski [8] [9]. According to Petroski, failures are subject to arise when engineers design structures that have no precedent which can be copied. For example, during the 19th century, iron bridges were collapsing from what later was identified at metal fatigue. Each time there was a failure, engineers would take note and determine why the failure occurred and how the failure could be obviated in future designs. The newer forms were thus, to some extent, an accumulation of the history of failures. These histories of failures show, he believes, that "engineers and designers ... learn much more from failures than from successes" (p. 1, [9]).

Petroski may well have proposed "form follows failure" as an alternative to the ubiquitous "form follows function". The two are worth comparing. "Form follows failure" suggests that engineers are frequently throwing backward glances to the past as they create their designs; "form follows function" alludes to a forward-thinking designer, carefully anticipating future needs. "Form follows failure" stresses the absence of negative features; "form follows functions" believes in the presence of positive attributes. Although "form follows failure" may well account for the current forms we see around us, "form 
follows function" has stronger, more positive connotations associated with it.

\subsection{Form Follows $X$}

There is a potentially long list of factors why product have taken on particular forms. These can be summarized as "Form Follows X". "X" refers to any one of these factors, such as "function", "manufacture", "assembly", "safety" and "cost" to name a few. Thus, the "Form Follows X" family echoes the design strategies of the "Design for $\mathrm{X}^{\prime}$ series of paradigms. The one notable exception is that of failure, for "Form Follows Failure" may hold true, while "Design for Failure" is not an advisable working paradigm.

\section{Meanings of Function}

When we say "form follows function", what do we mean by "function"? What is it that allows us to identify something as a function? What is it about function that allows us to attach a form to it?

\subsection{Function and Engineering}

In the engineering context, function is usually associated with what a object does: a bolt fastens, a truck transports, a furnace heats and a saw cuts. Form, on the other hand, is what the object is: a bolt, a truck, a furnace and a saw. If function is the verb, then the form is the noun.

Within design, however, function has important intentional qualities. A form may "do" many things, but only a select few would be referred to as functions. A reference to function implies that a particular action that the form performs is in accordance with the intent or will of the designer.

Sometimes the actions of a form are not in keeping with the designer's intent. We call this failure. To identify a form as failure, we must be able to distinguish between what a form does and what the designer wishes it to do. Function, described merely as what the form does, offers little discriminating power.

To make the distinction explicit, Gero [10] proposes the Function-Behaviour-Structure model of design. In this model, function refers to the intentional, purposeful or teleological qualities of the form. Behaviour is then reserved for what the form actually does. Structure is what the form is.

The insertion of behaviour allows us to consider a form's actions independently from the intent of the designer. The concept of failure does not apply for the behaviour is determined causally through inspection of the form. This causal connection indicates a uni- directional quality. In other words, it makes no sense to speak of determining the form from the behaviour.

Unfortunately, the separation of intent from behaviour is a difficult task for a number of reasons. First, if we wish to determine behaviour irrespective of intent, there are potentially an infinite number of behaviours I could describe for a given form. For instance, I could determine the behaviour of a refrigerator dropped from a height of $10 \mathrm{~m}$ over a frozen lake. Such description are, however, quite absurd as these circumstances are far from the context for which the refrigerator is designed. Hence, when describing behaviours, these descriptions are normally limited to closely coincide with the intent of the form. Second, Gero also talks about the actual behaviour and the intended behaviour. We identify particular levels of failure by particular levels of mismatch between the actual and intended behaviour. Intent is, once again, linked to behaviour.

Once presented with the concept of intended behaviour, how do we distinguish between intended behaviour and function? We can think of function as referring to intent on a grander scale than intended behaviour can hope to capture. Suppose I design a machine with a rotating brush to clean a surface. I build the machine and everything works as I intended. However, when I actually use the machine to clean the surface, the machine fails to remove the dirt as anticipated. The machine behaved as intended, but the function was left unfulfilled. I had misjudged the environment in which the machine was expected to operate. On a grander scale, we can consider the function of, say, the U.S. space shuttle. We could claim that the function of the space shuttle is to present the U.S. as a world leader. Intent on this scale is very difficult to relate to a structure of some kind. In other words, it would be impossible to determine this grand, overarching function based solely on the physical attributes of the space shuttle.

With the given terminology, the term "intended function" may seem redundant. However, I still believe the term has some meaning. Consider an adjustable wrench. We can say that the function of the wrench is to apply torque. I may own such a wrench but, over time, rust sets in, making the tool unadjustable. To make matters worse, it is rusted in the completely shut position. I find myself using it as a hammer. From my perspective, the function of the tool is to drive in nails. It cannot be to apply torque because it can grip nothing. An observer, unaware of the rust, may take exception to my use of the wrench, claiming that such usage is not in keeping with the intended function of the tool. Here, the use of the word "intended" stresses the intent of the designer over that of the user. 


\subsection{Function and Biology}

Biology has a different perspective on function than engineering. Engineering makes a strong link between function and intent and this link is what allows engineers to identify certain features as functions and, furthermore, the feature's functional aspects is how we explain its existence. In contrast, biology, favouring a more mechanical universe, seeks to break the link between function and intent. In so doing, the explanatory aspect of function that engineers take for granted is lost. How then, do biologists identify a particular feature of a form as a function, while other features are deemed "functionless"? When we say, "The function of the heart is to pump blood", what do we mean by function? What compels us to say the beating sound is not a function of the heart?

Wright [11] revisits and critiques some of the answers proposed for these questions. For instance, one may be inclined to state that pumping is a function and beating is not because "pumping" is useful to the system, whereas "beating" is not. In many cases, however, what constitutes the system and what is deemed "useful" remains unclear. Furthermore, a "useless" feature may still be deemed a function. Wright uses the example of a second hand on a watch. Even though he may find that particular feature to be useless, the function of the second hand is still to "read seconds more easily". (What is perhaps slightly odd here is that Wright, speaking from a biological perspective, uses an engineering example rather than a biological one. For example, one could claim that the function of reproductive organs is to produce offspring even if, for certain individuals, they are never used for that purpose.) To complicate matters still further, the beating of the heart can be useful as it allows for the diagnosis of some heart ailments. Nevertheless, beating remains a non-functional feature of the heart.

One might claim that the pumping feature of the heart is a function because it is necessary to pump blood, but there are other possible entities which may also be able to pump blood. In that case, pumping is a function of the heart because it is sufficient for pumping. Unfortunately, this line of reasoning fails to explain why the heart is there and not something else.

Cummins [12] believes that the problem arises because we mistakenly use the term "function" to explain the existence of a particular feature. Rather, we should use the term "function" to describe a capacity that a particular form has. Hence, pumping is a function of the heart because the heart has a capacity to pump blood.

Returning to the engineering domain, the biological explanation of the identification of function is not particularly satisfying. To say that a saw has a capacity to cut or a that a truck has a capacity to transport is not what compels engineers to to identify cutting and transporting as functions. When applied to engineering products, these functions retain strong intentional connotations.

\section{Linking Form to Function}

The preceding discussion surrounding some possible definitions of form and function were intended first, to show the complexity of the concepts and second, to hint at how the two are very much intertwined. I will now present a number of ways which I believe will make the connections between the two more explicit.

\subsection{The Logical Connection}

The axiom of "form follows function" alludes to a logical connection between form and function. It is the nature of this logic that has invited many a lively discussion.

The logic of design is often explained with reference to the philosophy of C.S. Peirce, presented both by March [13] and, later, by Coyne et al. [14]. Peirce proposes a triad of Case-Rule-Result. This particular order of the triad speaks of deduction: one begins with an observed case, applies the appropriate rule and deduces the result. Deduction is akin to causality and this is the basic approach that Gero refers to when he suggests that behaviour can be determined causally from a particular form. Deductive logic thus applies to the analytical side of design. The rules required to carry out deduction come from repeated observations of case and result, referred to as induction (Case-Result-Rule). Hence, induction is said to precede deduction. Within design, we use induction when we observe that a particular kind of form behaves in a repeatable way within a particular environment. We can associate this type of logic with design experience. The final triad is abduction. Abduction begins with a particular result, and then selects appropriate rules to determine which case is likely to have given rise to the result (ResultRule-Case). The case may be pre-existing, or it may be something completely original. Abduction is generally viewed as the logic that describes the synthetic design activity: the result is the desired functionality and the case is the designed form that will hopefully result in that functionality.

Deduction is generally considered to be the strongest, most reliable logic for it exemplifies convergent thinking, leading to a single ("correct") answer. Abduction is divergent, for there are many possible cases that can produce the same result. Thus, the logic of abduction is often portrayed as "weaker" than that of deduction and the logical link that joins 
form to function is also deemed to be "weak". All is not lost, however, for in terms of creativity, the bragging rights clearly belong to abduction.

\subsection{The Mathematical Connection}

Mathematics can be used to describe both the functional aspects of a product and its form, i.e., its shape. From a mathematical perspective, function refers to causal, non-intentional aspects of the form's behaviour. We use a mathematical equation, for example, to describe the force on a surface caused by wind, or the bending moment imposed on a beam. Mathematics also described the geometric features of the form. The geometric features are often used as parametric inputs into the equations that describe a form's behaviour. Thus, the bending moment depends on the geometry of the form subjected to the force.

The connection between mathematics and form is made more apparent when one considers manufacturing for equations can be used to describe the path a tool must take in a manufacturing process to produce the required form. As I suggested earlier, the operations that the equations describe can be viewed as a function within the domain of process planning. Thus, a single equation describes both a function and a form resulting from that function.

The geometry of a form is also used as a metaphor within design. Alexander [5] states that a welldesigned product must fit its environment. We can think of a hand fitting snugly into a glove. When we speak of fit in this sense, we are often referring to functional constraints. A noisy machine is not a good fit with a hospital environment. Electrical components may not be a good fit in a wet environment. Thus, we can understand functional appropriateness with reference to the geometry of a form.

\subsection{The Manufacturing Connection}

Manufacturing has had a significant influence on how engineers view form and function within the design arena. The influence may be attributable to the ambiguity of form, for a mechanical designer and a process planner may look at the same form with very different eyes.

The influence of manufacturing on the design process is evident with the development of manufacturing engineering. As a legitimate branch of engineering, this relatively new area of study attests to the need to include manufacturing concerns in design. If mechanical designers assign form to their functional requirements and have little knowledge of manufacturing processes, they may ask the designated manufacturers of that form to perform difficult, unnecessarily costly or even impossible tasks. Manufacturing methods may impose limits on the possible forms which, in turn, may lead to modification of the functional requirements. By bringing mechanical designers and process planners together, these difficulties can be largely avoided, giving the design activity greater coherence.

The axiom that "form follows fabrication" refers to the belief that the form of a product should reflect the manufacturing process that gave rise to it. A machinemade product should look machine-made; a handmade product should look hand-made. The manufacturing process can thus imbue the product with an additional "feature". We can refer to this feature as a "high-level" function, similar to the "political" function of the space shuttle as promoting the U.S. as a world leader. In the earlier days of the manufactured good, these added features gave the user/owner sense of status, for that "machine-made" appearance, made possible by the latest technology, connoted modernity and progress. In this way, we might claim that the manufacturing process can add "function" to the product. (The added "function" can also be apparent. Many have noted that aluminum foil has one side shinier than the other. Others are quick to point out that the difference in sheen is due to the manufacturing process, not the intent of the designer. Nevertheless, the question often arises, when cooking, is it better to have the shiny side facing inward or outward?)

Machines used in manufacturing owe their existence to engineering design. These machines, in turn, bring new possibilities for yet newer designs. Historically, for instance, it was assumed that if one wished to make money by manufacturing products, one would cater to the rich, for it is they who could most afford to buy. With new manufacturing techniques and the rise of mass-production, it became possible to make a profit selling goods to the poor [15]. This new market invited a new line of products, with new forms to be designed and new functionalities to be conceptualized.

The more subtle way that manufacturing connects form to function is through process planning as I suggested in the introduction. The form, a concrete entity to the mechanical designer, becomes abstract as the process planner imagines all the possible machining functions that might result in that form. Manufacturing thus invites mechanical designers to see the form as abstract and the function as concrete.

\subsection{The Nature Connection}

Nature is often seen as a source of inspiration for both form and function. For some, nature embodies perfection, and therefore worthy of reproduction.

Thompson [16] looks at form from a biological perspective. He believes that the "form of an object is 
a 'diagram of forces'" (p. 11). These forces, either past (for solid forms) or present (for liquid forms) are imposed on the forms by the natural environment. These forces alter the shape of the form. There is yet another important shape-changing influence, namely that associated with growth. This growth can be seen to generate a series of curves, such as in the shells of some sea animals and these curves echo mathematical equations. Mathematics once again provides a strong link, this time relating forms in nature with those in the human-built world.

Nature is also understood with reference to functionality, despite the fact that function raises questions concerned with intent as discussed earlier. The function we see in nature is reflected in many of our designs (or, conversely, we see function in nature because we saw it first in our own designs). A common example is that of velcro, invented by de Mestral after studying Burdock seeds [17]. Not only was this a new form, but it extended the engineering concept of the function "fastening". On a perhaps grander scale, LeCorbusier saw parallels between the human body and the house [18]: a house has ventilation, electricity, a sewer system, and allows for the circulation of people while the body has a respiratory system, a digestive system, a nervous system and a circulatory system. The house, like a tool, becomes an extension of the human body. The two examples of velcro and the house differ in distinct ways. Velcro is normally an individual component; a house is a system. Velcro mimics the Burdock seed more in form than in function; the house mimics the human body more in function than in form.

\subsection{The Language Connection}

The concepts of form and function lend themselves readily to a language metaphor. The function is about doing (verb) and the form is about being (noun). One may presumably attach other parts of speech to other features of design.

There is perhaps a richer language metaphor that can be applied to design. This metaphor arises if we posit an engineering product catalogue as a dictionary. The components in the catalogue are the words of the dictionary. The components can be put together in particular fashions to create the desired functionality, just as words can be strung together in certain ways (i.e., according to rules of grammar) to create the desired meaning. The forms and functions of design parallel the words and meanings of language.

The dictionary works well if I have an unknown word and I wish to know its meaning; the catalogue works well if I have a component and I wish to know its function (specifications and performance characteristics). Both of these tasks are quite straightforward as they follow deductive, convergent reasoning. If, however, I wish to proceed in the opposite direction (abductive reasoning), my task becomes much more difficult. In other words, suppose I have a meaning and I wish to locate the corresponding word or, equivalently, I have the function and wish to find a suitable component. In language, I may resort to a thesaurus; in design, the resources are quite limited.

The language metaphor suggests yet another strong connection between form and function. One of the peculiar features of dictionaries is that they define words using words. Thus, a dictionary is one large tautology: if I find the meaning of a given word and look up the words that are in the definition of that word and continue looking up words in those definitions, I could find myself back where I started, with the original word. Words and meanings become inseparable. If the language metaphor holds, form and function may also be inseparable. The implication is that, in order to understand what a function is, one must be able to refer to at least one form that embodies that function. As the number of forms in a functional class increases, ones understanding of the function increases with it. Our concept of function is thus dependent on our knowledge of forms.

\section{Summary and Conclusions}

Process planning invites engineers to reconsider their conception of the design process. Process planners select functions to create the desired form; mechanical designers select forms to create the desired function. The "interchangeability" of form and function may have implications within the large field of engineering design.

Definitions of "form" and "function" reveal some of the potential links between them. The definition of "form" is fairly straightforward although the use of the word in computer science or software engineering strongly suggests that "form" need not or should not be used exclusively for physical objects.

Definitions of "function" are much more problematic. Difficulties arise when due to intentionality implicit in the term. The definitions of "function" vary according to the level of intentionality assigned to the term, from essentially none (as in biology) to a great deal (as in the realization of political goals). The intentionality of engineering design and the need to analyse the designed product prompt us to separate out the intent of a particular form from its mechanical actions, designated its behaviour. Detached from intent, the behaviour of a product can theoretically be "deduced" in a causal-like fashion by inspection of the form. In practice, however, we must limit the number of behaviours 
analysed to those deemed relevant, i.e., as defined by the designer's intent. Thus, the link joining behaviour to intent can never be fully severed.

Although the idealized form of the engineering design process seeks to separate function (as abstract) from form (as concrete), we have reason to believe that they should be linked together.

Logic links form to function but imposes directional properties on the connection: one can deduce function from form ("strong" logic) but only abduce form from function ("weak" logic). The deductive logic forces us to remove most of the intentional qualities from "function" (thus "function" is better rendered "behaviour").

Engineers apply mathematics liberally, whether for the form or the function. Mathematics can describe the geometry of the form or the causal link of the function. Used to define a tool path in manufacturing, a single mathematical formula unites function (the machining operation) with form (the resulting shape of the machined object).

Manufacturing connects form to function on several fronts. First, manufacturing concerns invite a broadening of our understanding of design beyond the final drawings. Second, as new manufacturing techniques are introduced, new possibilities of both form and function arise. Third, process planning invites mechanical designers to view form as an abstract concept.

Nature presents engineers with idealized functions embodied by seemingly perfect forms. By imitating nature, engineers echo the strong form-function connection.

Language reminds us that form and function, like words and meanings, are essentially inseparable. Language also demonstrates why we should not expect to move from function to form as easily as from form to function.

By highlighting the difficulties of defining form and function and by demonstrating a myriad of connections between them, I hope to draw attention to, first, the problems associated with the creation of (computerized) design tools relying on distinctions of form and function and, second, to suggest ways that the software might be made more effective for a wide range of potential users.

\section{References}

[1] Rindele, J.R., E.R. Colburn, S.P. Hoover, J.P. PazSoldan, and J.D. Watton. "Form-Function Characteristics of Electro-Mechanical Designs". In Newsome, S.L., W.R. Spillers and S. Finger (eds.). Design Theory '88: Proceedings of the 1988 NSF Workshop on Design Theory and Methodology. Springer-Verlag, New York, 1988.
[2] Longenecker, S.N. and P.A. Fitzhorn. "Form + Function +Algebra = Feature Grammars". In Newsome, S.L., W.R. Spillers and S. Finger (eds.). Design Theory '88: Proceedings of the 1988 NSF Workshop on Design Theory and Methodology. Springer-Verlag, New York, 1988.

[3] Yellowley, I. and P. Winkelman, "A simple proposal for the development of Intelligent Design Catalogs", presented at the Second CDEN International Conference on Design Education, Innovation, and Practice, Kananaskis, Alberta, July, 2005.

[4] Pye, D. The Nature of Design. Studio Vista, London, 1964.

[5] Alexander, C. Notes on the Synthesis of Form. Harvard University Press, Cambridge, Massachusetts, 1967.

[6] http://en.wikipedia.org/wiki/Form_follows_function. Accessed March 2, 2007.

[7] Bill, M. Form. Verlag Karl Werner, Basel, 1952.

[8] Petroski, H. To Engineer Is Human: The Role of Failure in Successful Design. Vintage Books, New York, 1992.

[9] Petroski, H. Design Paradigms: Case Histories of Error and Judgment in Engineering. Cambridge University Press, New York, 1994.

[10] J.S. Gero, K.W. Tham and H.S. Lee, "Behaviour: a link between function and structure in design", in D.C Brown, M. Waldron and H. Yoshikawa (eds.), Intelligent Computer Aided Design, North Holland (Elsevier), Amsterdam, pp. 193-220, 1992.

[11] Wright, L. "Functions". In Buller, D.J. (ed.), Function, Selection and Design. State University of New York Press, Albany, NY, 1999, pp. 29-55.

[12] Cummins, R. "Functional Analysis". In Buller, D.J. (ed.), Function, Selection and Design. State University of New York Press, Albany, NY, 1999, pp. 57-83.

[13] March, L.J. "The Logic of Design". In Cross, N. (ed.), Development in Design Methodology, Wiley, Chichester, 1984.

[14] Coyne, R.D., M.A. Rosenman, A.D. Radford, M. Balachandran, J.S. Gero. Knowledge-based design Systems. Addison-Wesley Publishing Company, Reading, MA, USA, 1990.

[15] von Mises, L. http://www.fff.org/freedom/0993e.asp, 1993 (accessed June 12, 2007).

[16] Thompson, D.W. On Growth and Form. Cambridge University Press, Cambridge, 1961.

[17] http://en.wikipedia.org/wiki/Velcro, 2007 (accessed June 12, 2007).

[18] Steadman, P. The evolution of Designs: Biological Analogy in Architecture and the Applied Arts. Cambridge University Press, New York, 1979. 